\title{
MEASUREMENT OF THE SPIN-ORBIT MISALIGNMENT OF KOI-13.01 FROM ITS GRAVITY-DARKENED KEPLER TRANSIT LIGHTCURVE
}

\author{
JASON W. BARNES ${ }^{1,5}$, Ethan LinSCOTT ${ }^{2}$, AND Avi SHPORER Sh, $^{3,4}$ \\ ${ }^{1}$ Department of Physics, University of Idaho, Moscow, ID 83844-0903, USA; jwbarnes@uidaho.edu \\ 2 Department of Physics, Oklahoma Baptist University, Shawnee, OK 74804, USA \\ ${ }^{3}$ Department of Physics, University of California, Santa Barbara, CA 93106, USA \\ ${ }^{4}$ Las Cumbres Observatory, Global Telescope Network, Santa Barbara, CA 83117, USA \\ Received 2011 August 2; accepted 2011 October 5; published 2011 October 31
}

\begin{abstract}
We model the asymmetry of the KOI-13.01 transit lightcurve assuming a gravity-darkened rapidly rotating host star in order to constrain the system's spin-orbit alignment and transit parameters. We find that our model can reproduce the Kepler lightcurve for KOI-13.01 with a sky-projected alignment of $\lambda=23^{\circ} \pm 4^{\circ}$ and with the star's north pole tilted away from the observer by $48^{\circ} \pm 4^{\circ}$ (assuming $M_{*}=2.05 \mathrm{M}_{\odot}$ ). With both these determinations, we calculate that the net misalignment between this planet's orbit normal and its star's rotational pole is $56^{\circ} \pm 4^{\circ}$. Degeneracies in our geometric interpretation also allow a retrograde spin-orbit angle of $124^{\circ} \pm 4^{\circ}$. This is the first spin-orbit measurement to come from gravity darkening and is one of only a few measurements of the full (not just the sky-projected) spin-orbit misalignment of an extrasolar planet. We also measure accurate transit parameters incorporating stellar oblateness and gravity darkening: $R_{*}=1.756 \pm 0.014 \mathrm{R}_{\odot}, R_{\mathrm{p}}=1.445 \pm 0.016 \mathrm{R}_{\mathrm{Jup}}$, and $i=85.9 \pm 0.4$. The new lower planetary radius falls within the planetary mass regime for plausible interior models for the transiting body. A simple initial calculation shows that KOI-13.01's circular orbit is apparently inconsistent with the Kozai mechanism having driven its spin-orbit misalignment; planet-planet scattering and stellar spin migration remain viable mechanisms. Future Kepler data will improve the precision of the KOI-13.01 transit lightcurve, allowing more precise determination of transit parameters and the opportunity to use the Photometric Rossiter-McLaughlin effect to resolve the prograde/retrograde orbit determination degeneracy.
\end{abstract}

Key words: eclipses - stars: individual (KOI-13) - techniques: photometric

Online-only material: color figures

\section{INTRODUCTION}

The Sun's planets all orbit in planes that vary in orbital inclination from one another by just $\sim 1^{\circ}-7^{\circ}-$ a situation that supports the nebular hypothesis for the solar system's origin (see, e.g., Lissauer 1993). The orbital planes all differ by between $\sim 3^{\circ}$ and $7^{\circ}$ from the Sun's equatorial plane, and by $\sim 0.3-6.3$ from the invariable plane (the plane normal to the net solar system angular momentum vector). Although angular distances are typically provided for these differences, we think that they are better shown in two dimensions. Figure 1 shows the location of the orbital angular momentum vectors for various solar system objects in ecliptic coordinates.

Given its utility in constraining the origin of the solar system, measurement of the mutual inclination between extrasolar planets' orbits and/or the inclination of extrasolar planets' orbit normal relative to their stars' spin axis-“"spin-orbit alignment," $\varphi$ - can shed light on the origin of those extrasolar systems as well. Evidence so far shows that while the solar system is not unusual, a wide variety of planetary system types exists.

Some systems have planets that are even more nearly coaligned with each other than those in the solar system. In particular, the Kepler-11 six-planet system has typical mean mutual inclinations of just $1^{\circ}-2^{\circ}$ (Lissauer et al. 2011). As evidenced by Figure $1, \mathrm{a} 1^{\circ}-2^{\circ}$ mutual inclination is significantly smaller than the mutual inclination of the solar system's terrestrial planets. Interestingly, however, the solar system giant planets have mutual inclinations of $0.7-2.0$, comparable to those in the Kepler- 11

\footnotetext{
5 Researcher ID: B-1284-2009
}

system. We think that this similarity in mutual inclinations between the solar system giants and the Kepler-11 planets may indicate similar formation and evolution, but distinctly different from that of the solar system terrestrial planets. Although coplanarity of a system's planets and a planet's spin-orbit alignment are distinct measurements, they share the property that the proposed mechanisms for misaligning one will likely misalign the other as well.

Radial velocity measurements of planet-hosting stars during transits can indicate spin-orbit alignment via the Rossiter-McLaughlin effect. Rossiter-McLaughlin observations have revealed that a majority of close-in extrasolar planets appear to be spin-orbit aligned (20 of 31 measurements of $\lambda$ tabulated by Perryman 2011, p. 129 , are within $2 \sigma$ of zero). For example, Winn et al. (2006) showed that HD189733 has a spin-orbit misalignment of $1.4 \pm 1.1$, which Triaud et al. (2009) later refined to just $0.85 \pm 0.30$. HAT-P-1's measured inclination of $3.7 \pm 2.1$ is consistent with spin-orbit alignment (Johnson et al. 2008). We note that, based on Figure 1, each of these planets is more closely spin-orbit aligned than is Jupiter, which has an inclination of 6.1 with respect to the Sun's equatorial plane. Many more planets have been determined to be spin-orbit aligned to lower precision (e.g., Simpson et al. 2010, 2011; Miller et al. 2010; Hellier et al. 2011, to name a few recent measurements).

On the other hand, Rossiter-McLaughlin observations have also revealed a significant population of spin-orbit misaligned planets. For instance, HAT-P-30b is misaligned by $74^{\circ} \pm 9^{\circ}$ (Johnson et al. 2011) and XO-3 is misaligned by $70^{\circ} \pm 15^{\circ}$ (Hébrard et al. 2009) (see Perryman 2011, 


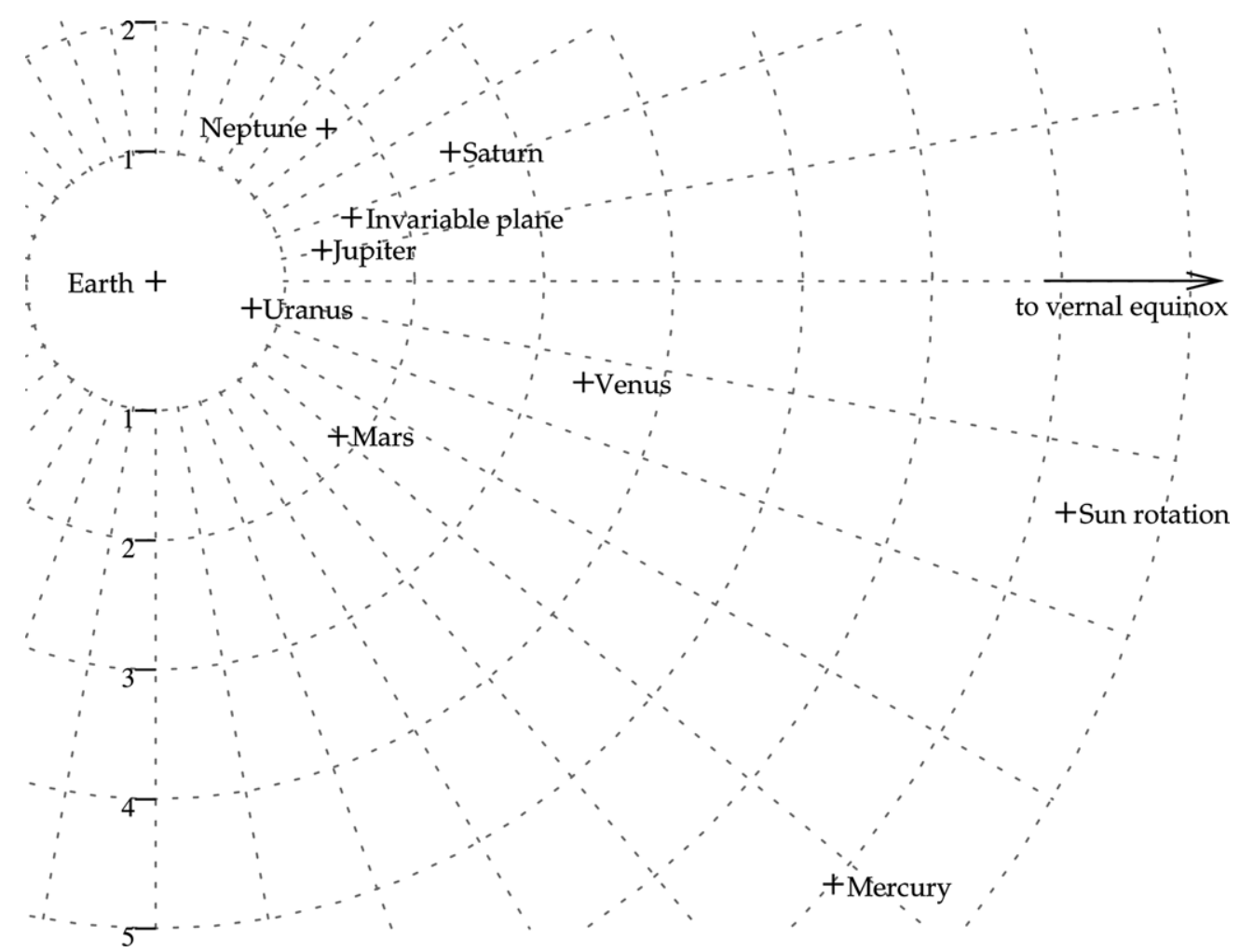

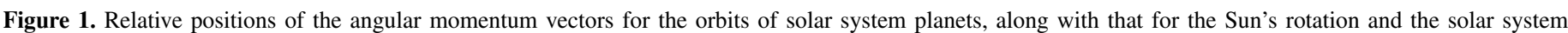

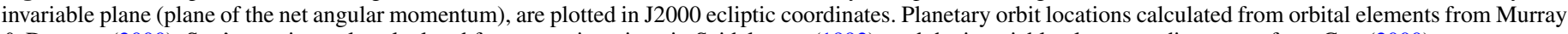
\& Dermott (2000), Sun's rotation pole calculated from equation given in Seidelmann (1992), and the invariable plane coordinates are from Cox (2000).

Table 6.1 for a summary of spin-orbit alignments). Many planets are even retrograde, such as HAT-P-11b (Winn et al. 2010b; Hirano et al. 2011), WASP-17b (Anderson et al. 2010), and HAT-P-7b (Narita et al. 2009; Winn et al. 2009b). Winn et al. (2010a) noted that these highly spin-orbit-misaligned planets occur preferentially around hot stars, implying that planet formation and/or evolution differs around more massive stars.

The Rossiter-McLaughlin effect has also been used to determine the spin-orbit alignment of stellar binaries (Albrecht et al. 2007, 2009, 2011). In fortuitous cases, a star shows spot activity in the area under which the planet transits. Those spots' effects on the transit lightcurve can then be used to constrain spin-orbit alignment (Sanchis-Ojeda et al. 2011; Sanchis-Ojeda \& Winn 2011; Nutzman et al. 2011).

Barnes (2009) showed that the spin-orbit alignment for planets orbiting rapidly rotating stars can be determined from transit photometry alone, taking advantage of the nonuniformity of those stars' disks (von Zeipel 1924). The nonuniformity introduces characteristic asymmetries into planets' transit lightcurves. The photometric technique described by Barnes (2009) has an advantage over Rossiter-McLaughlin measurements in that it can measure both the longitude of the planet's ascending node and the stellar obliquity to the plane of the sky. As a purely radial measurement, Rossiter-McLaughlin determines only $\lambda$, the projected spin-orbit alignment. The Rossiter-McLaughlin-measured spin-orbit alignment distribution must be deprojected in order to ascertain the true distribution (Fabrycky \& Winn 2009).

Recently, Szabó et al. (2011) found an asymmetry in the Kepler transit lightcurve of KOI-13.01 that they attributed to spin-orbit misalignment around a fast-rotating star, consistent with the Barnes (2009) predictions. In this paper, we use this asymmetry to measure the spin-orbit misalignment angle $\varphi$ of the KOI-13.01 system using the Barnes (2009) model. The present work, focused on the measurement of $\varphi$, leaves to others the analysis of the complete lightcurve and secondary eclipse. In Section 2, we describe data reduction of the Kepler public Q2 lightcurve for this planet, and we describe the gravity-darkened rapidly rotating star transit lightcurve model and its application in Section 3. We then show the results of our fit in Section 4 and discuss the implications of the measurement in Section 5. We give our concluding remarks in Section 6.

\section{OBSERVATION}

The Kepler mission (Borucki et al. 2010) science team first identified a planet candidate around the star with designation 9941662 in the Kepler Input Catalog (KIC; Brown et al. $2011)$ in the first two quarters of science data (Q1 and Q2; Borucki et al. 2011). A discovery paper confirming the resulting Kepler Object of Interest (KOI) KOI-13.01 as a planet is presently in preparation (Rowe et al. 2011). The KIC records the star's location as $19.13141 \mathrm{H}+46.8684$, and provides stellar parameters of $M_{*}=1.83 \mathrm{M}_{\odot}, T_{\text {eff }}=8848 \mathrm{~K}$, and a magnitude in the Kepler bandpass of $K_{p}=9.958$ (Borucki et al. 2011). Later additional ground-based spectroscopy by Szabó et al. (2011) determined $M_{*}=2.05 \mathrm{M}_{\odot}, T_{\mathrm{eff}}=8511 \pm 400 \mathrm{~K}$, and a $\Delta$ magnitude between KOI-13 and its companion of 0.29 . Szabó et al. (2011) also measured $v \sin i=65 \pm 10 \mathrm{~km} \mathrm{~s}^{-1}$ for KOI-13.

We use the Kepler photometry, in which apparent transit depth for KOI-13.01 is different in the Q0, Q1, and Q2 Kepler time series. This interquarter variability (as well as the intraquarter variability within Q1) derives from use of a too-small aperture in Q1 that did not gather all of the flux from this saturated bright 
star. Therefore for our investigation we, like Szabó et al. (2011), use just the Kepler Q2 public domain photometry, downloaded from the Multimission Archive at STScI (MAST). We use the Q2 short cadence data in our analysis.

In reducing the data first we eliminate data with imperfect trend subtractions that were acquired near data gaps, leaving 111,858 one-minute measurements in the short cadence time series. Then we clip out the points whose flux varies by more than $5 \sigma$ away from the six surrounding points - this eliminates 176 bad measurements. With no evidence of transit timing variations or other transit-to-transit variability, we then fold the data using the Borucki et al. (2011) period of 1.763589 days. We crop the time series around the transit itself so as not to consider the interesting effects in the lightcurve other than the transit (i.e., Shporer et al. 2011b). In order to speed computation time in fitting, we bin the data into $60 \mathrm{~s}$ bins, typically coadding 45 points bin $^{-1}$ and leaving 254 points in the timeseries with characteristic precision of $28 \mathrm{ppm}$.

Finally, we subtract off $45 \%$ of the median flux in order to compensate for light from KOI-13 's binary companion polluting the photometry (following Szabó et al. 2011).

\section{MODEL}

We generate model lightcurves using the algorithm first described in Barnes \& Fortney (2003), suitably modified for gravity-darkened rotating stars by Barnes (2009). It has four different methods to calculate transit fluxes. In order of increasing accuracy and required computation time, they are: analytical (after Mandel \& Agol 2002, with gravity-darkening modifications), image-pixel-based cartesian, radial onedimensional numerical integration (which we call "polar"), and radial/azimuthal two-dimensional numerical integration (which we call "full polar").

For this work we also added the following features to the code.

1. Fit for $\psi, \lambda$. We added the ability to fit for the stellar obliquity, $\psi$, and for the longitude of the ascending node of the planet's orbit, $\omega$. In this paper, we express $\omega$ in terms of $\lambda$, the sky-projected spin-orbit angle, in order to provide comparability with existing measurements of other transits from the Rossiter-McLaughlin effect (e.g., Perryman 2011 , p. 128). Hence, we define $\lambda$ to be the longitude of the ascending node as measured counterclockwise from the ascending node of the star's rotational equator. Given the Szabó et al. (2011) measurement of stellar $v \sin i$ (which is $v \cos \psi$ using our parameters), we therefore couple the stellar rotation rate $\Omega_{*}$ to the stellar obliquity such that:

$$
\Omega_{*}=\frac{(v \sin i)_{\text {measured }}}{R_{*} \cos \psi}
$$

2. Time integration. When fitting data, for each model photometric data point we numerically integrate the flux for $\pm t / 2$ around the central time for the point, where $t$ is that point's integration time. This has little effect on the short cadence data, but greatly improves the accuracy of fits using the 30 minute effective integration time for Kepler's long cadence data (Kipping 2010).

3. Bandpass. We modified our algorithm to integrate flux numerically across the published Kepler response function. ${ }^{6}$ Wavelength effects are incorporated entirely into

\footnotetext{
6 http://keplergo.arc.nasa.gov/CalibrationResponse.shtml
}

limb-darkening parameters for non-rotating stars. But fastrotators' temperature heterogeneity introduces variations in transit lightcurve shape as a function of wavelength (Barnes 2009). In practice, for KOI-13, on which we fix the polar temperature to be $8848 \mathrm{~K}$ (the value from the KIC), the effects of the bandpass are nearly indistinguishable from a monochromatic model at $0.5934 \mu \mathrm{m}$.

4. Fit for $M_{*}$. Transit lightcurves for non-rotating stars can constrain only the stellar density, $\rho_{*}=3 M_{*} / 4 \pi R_{*}^{3}$. They cannot determine the radius $R_{*}$ and mass $M_{*}$ individually because, as can be derived from Kepler's law, $M_{*} / R_{*}^{3}=$ $1 / G T_{\text {dur }}^{3} n$, where $G$ is the universal gravitational constant, $T_{\text {dur }}$ is the transit duration, and $n$ is the planet's orbital mean motion (which is set by the orbital period). Since $G, T_{\text {dur }}$, and $n$ are all constant, there are no independent constraints on $M_{*}$ or $R_{*}$ individually. For a fast-rotating star, however, the intensity of the gravity darkening effect depends on the local effective gravity. For instance, the ratio of the equatorial stellar effective temperature $T_{\text {eq }}$ to the polar temperature $T_{\text {pole }}$ is

$$
\frac{T_{\mathrm{eq}}^{4}}{T_{\mathrm{pole}}^{4}}=\frac{R_{\mathrm{p}}^{2}}{R_{*}^{2}}\left(1-\frac{\Omega^{2} R_{\mathrm{eq}}^{3}}{G M_{*}}\right) .
$$

The $\left(R_{\text {eq }}^{3} / M_{*}\right)$ term provides no new constraints, of course. But $\Omega$ depends only on $R_{*}$, and not on $M_{*}\left(\left(R_{\mathrm{p}} / R_{*}\right)\right.$ is also a function of $\Omega$-see Seager \& Hui 2002 for how they relate in a point-core model relevant for stellar moments of inertia). So, gravity darkening can be used to break the stellar density degeneracy.

In practice, however, this is a weak constraint. A stellar mass of $M_{*}=0.001 \mathrm{M}_{\odot}$ can be immediately (if unhelpfully) ruled out for KOI-13 because even with zero obliquity $\left(\psi=0^{\circ}\right)$ it would need to be rotating faster than its breakup speed to achieve $v \sin i=65 \mathrm{~km} \mathrm{~s}^{-1}$. Conversely, an $M_{*}=100 \mathrm{M}_{\odot}$ star would need to rotate with a sufficiently long period $P_{\text {rot* }}=2 \pi / \Omega$ that its gravity darkening would be negligible. We can derive lightcurve constraints on $M_{*}$ then because low-mass stars have exaggerated gravity darkening, and high-mass stars have muted gravity darkening. Either can then fail to fit the real lightcurve if the gravity darkening differs sufficiently from that of the real star. Highly asymmetric lightcurves better constrain $M_{*}$ on the high end, while more nearly symmetric lightcurves like that for KOI-13.01 constrain the low-mass end more effectively.

Lacking empirical constraints, we fix the gravity-darkening parameter $\beta$ when we fit the lightcurves. We use two different assumptions for the value of $\beta$ : the theoretical value from von Zeipel (1924) of $\beta=0.25$ and the experimental value of $\beta=0.19$ from Monnier et al. (2007). As expected, higher values of $\beta$ lead to more intense gravity darkening holding all other parameters equal. The ultimate effect on the transit lightcurve of changing $\beta$, therefore, is roughly similar to that of varying $M_{*}$ in that both primarily affect the intensity of gravity darkening. We found, however, that the best-fit values varied less than $1 \sigma$ when using $\beta=0.19$ rather than $\beta=0.25$. We therefore use the theoretical value of $\beta=0.25$ in our reported fits. More precise photometry and a better determination of the star's mass could therefore help to constrain $\beta$ empirically in the future.

We fit the model described above to the data using the Leavenberg-Marquardt algorithm described in Press et al. 
Table 1

Best-fit Transit Parameters for the KOI-13.01 System

\begin{tabular}{lcc}
\hline \hline Parameter & \multicolumn{2}{c}{ Best-fit Values } \\
\cline { 2 - 3 } & $M_{*}=1.83 \mathrm{M}_{\odot}$ & $M_{*}=2.05 \mathrm{M}_{\odot}$ \\
\hline$\chi_{\text {reduced }}^{2}$ & 1.409 & 1.419 \\
$R_{*}$ & $1.694 \pm 0.013 \mathrm{R}_{\odot}$ & $1.756 \pm 0.014 \mathrm{R}_{\odot}$ \\
$R_{\mathrm{p}}$ & $1.393 \pm 0.015 \mathrm{R}_{\mathrm{Jup}}$ & $1.445 \pm 0.016 \mathrm{R}_{\mathrm{Jup}}$ \\
$R_{\mathrm{p}}$ & 0.084508 & 0.084513 \\
$R_{*}$ & $85.9 \pm 0.4$ & $85^{\circ} .9 \pm 0.4$ \\
$b$ & 0.31962 & 0.31598 \\
$c_{1}$ & $0.49 \pm 0.03$ & $0.48 \pm 0.03$ \\
$T_{0}$ & $4628032 \pm 3 \mathrm{~s}$ & $4628033 \pm 3 \mathrm{~s}$ \\
$\lambda$ & $24^{\circ} \pm 4^{\circ}$ & $23^{\circ} \pm 4^{\circ}$ \\
$\psi$ & $-45^{\circ} \pm 4^{\circ}$ & $-48^{\circ} \pm 4^{\circ}$ \\
$\varphi$ & $54^{\circ} \pm 4^{\circ}$ & $56^{\circ} \pm 4^{\circ}$ \\
$P_{\text {rot } *}$ & $22.5 \mathrm{hr}$ & $22.0 \mathrm{hr}$ \\
$f_{*}$ & 0.018 & 0.021 \\
\hline
\end{tabular}

Notes. The time of inferior conjunction, $T_{0}$, is measured in seconds after BJD 2454900, after Borucki et al. (2011). There is a four-way degeneracy for the transit geometry, i.e., the projected spin-orbit angle $\lambda$ and the stellar obliquity $\varphi$-see Figure 3 . The stellar rotation period is denoted as $P_{\text {rot* }}$. The stellar dynamical oblateness is denoted as $f_{*}$.

(2007) to efficiently zero in the best parameters. Lacking appropriate analytical partial derivatives for most parameters in the gravity-darkened case (though see also Pál 2008, for analytical derivatives in the spherical star case), we calculate numerical partial derivatives with respect to each parameter at each point in each step.

To calculate the one-standard deviation $(1 \sigma)$ errors on each measurement, we follow a modified version of the set of steps specified by Press et al. (2007, p. 815). Instead of using the covariance matrix, we explicitly fit the data using a set of fixed values for each parameter. We then identified the minimum in $\chi^{2}$ as a function of each parameter. We assign the error for that parameter based on how far from the minimum value the best-fit $\chi^{2}$ increases by the appropriate amount given a $1 \sigma$ confidence level and our number of parameters of interest (8). This $\Delta \chi^{2}=9.304$ in our case, since we held $M_{*}$ fixed when fitting other parameters (see Section 4).

\section{RESULT}

We fit the Kepler Q2 short-cadence KOI-13.01 transit lightcurve for nine different parameters:

1. the stellar radius, $R_{*}$;

2. the planetary radius, $R_{\mathrm{p}}$;

3. the orbital inclination relative to the plane of the sky, $i$, also expressed as the impact parameter, $b$;

4. a single limb-darkening parameter, $c_{1}$, equal to the sum of the two quadratic limb-darkening parameters such that $c_{1}=u_{1}+u_{2}$, after Brown et al. (2001);

5. the time of inferior conjunction, $T_{0}$, which can be shifted relative to the mid-transit time for oblate stars (Barnes 2009);

6. the out-of-transit star flux, $F_{0}$;

7. the stellar mass, $M_{*}$;

8. the longitude of the planet's ascending node, $\lambda$, relative to that of the ascending node of the stellar equator; and

9. the stellar obliquity, $\psi$, measured as the tilt of the stellar north rotational pole toward Earth relative to the plane of the sky.

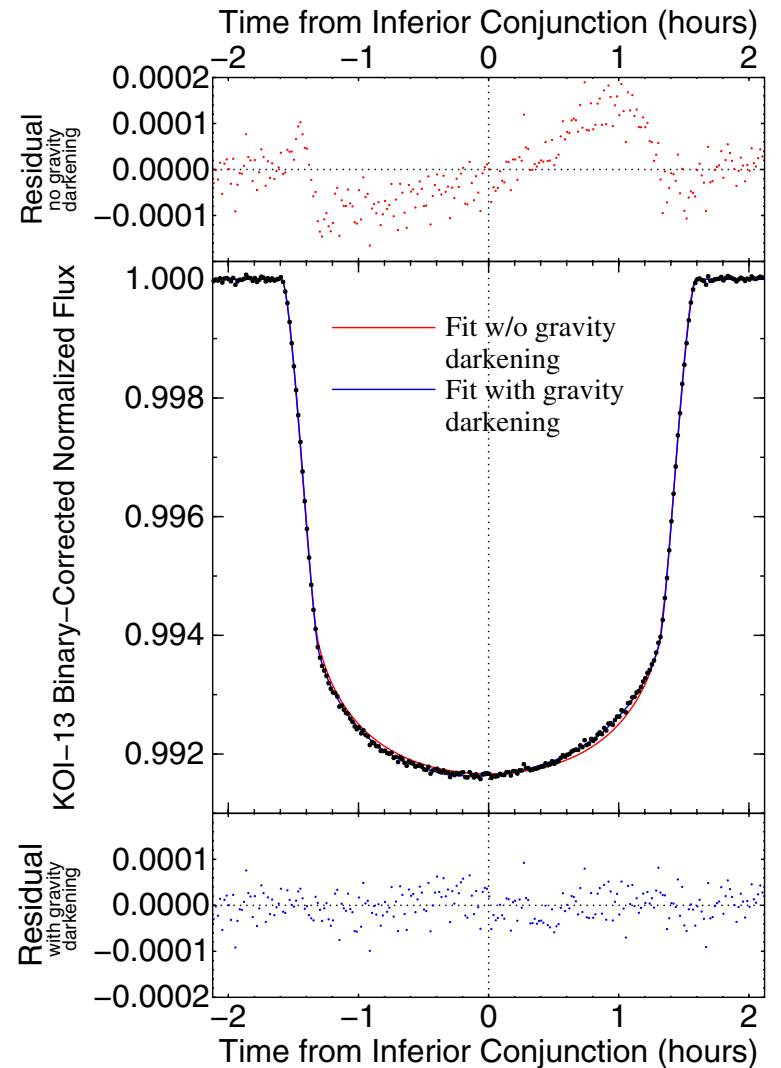

Figure 2. This plot shows the transit lightcurve for KOI-13.01 in filled black circles in the middle, with a best-fit line using gravity darkening $\left(M_{*}=\right.$ $2.05 \mathrm{M}_{\odot}$ ) in blue and one without gravity darkening in red. The size of the data circles corresponds to their error (as reported in the MAST, adjusted for binning) in the $y$-dimension (flux). We plot the residuals for the gravity-darkened fit as blue dots in the bottom graph. The residuals for the non-gravity-darkened fit are at top, as red dots.

(A color version of this figure is available in the online journal.)

We show the best-fit values along with their $1 \sigma$ uncertainties in Table 1. The fit itself along with the data is plotted in Figure 2. We also show in Figure 2 the best-fit lightcurve without gravity darkening, after Szabó et al. (2011), in order to draw attention to the necessity of the present analysis. The no-gravity-darkening fit fails to match the KOI-13.01 transit ingress and egress. It also does not adequately model the transit bottom, which for KOI-13.01 is darker in the first half of the transit than it is in the second half. The gravity-darkened model fits all of these characteristics - the ingress, egress, and asymmetric transit bottom.

The best-fit value for stellar mass $M_{*}$ is highly uncertain due to the weak dependence of gravity darkening on $M_{*}$ as discussed in Section 3. Both the Borucki et al. (2011) and Szabó et al. (2011) spectroscopic measurements of $M_{*}, M_{*}=1.83 \mathrm{M}_{\odot}$ and $M_{*}=2.05 \mathrm{M}_{\odot}$ respectively, lie within $2 \sigma$ of our measured value of $M_{*}=0.9 \pm 0.6 \mathrm{M}_{\odot}$. Our measurement clearly prefers lower values for $M_{*}$. However, the best-fit value of $M_{*}=0.9 \mathrm{M}_{\odot}$ is clearly inconsistent with the star's spectral type. Given the uncertainty in our measurement, however, for the remainder of the fitted parameters we show values for an assumed mass of both $M_{*}=1.83 \mathrm{M}_{\odot}$ (Borucki et al. 2011) and $M_{*}=2.05 \mathrm{M}_{\odot}$ (Szabó et al. 2011).

The reduced $\chi^{2}\left(\chi_{\text {reduced }}^{2}\right)$ values for each of those fits are $\chi_{\text {reduced }}^{2}=1.409\left(M_{*}=1.83 \mathrm{M}_{\odot}\right)$ and $\chi_{\text {reduced }}^{2}=1.419$ $\left(M_{*}=2.05 \mathrm{M}_{\odot}\right)$. We think that the $\chi_{\text {reduced }}^{2}$ above 1.0 results 


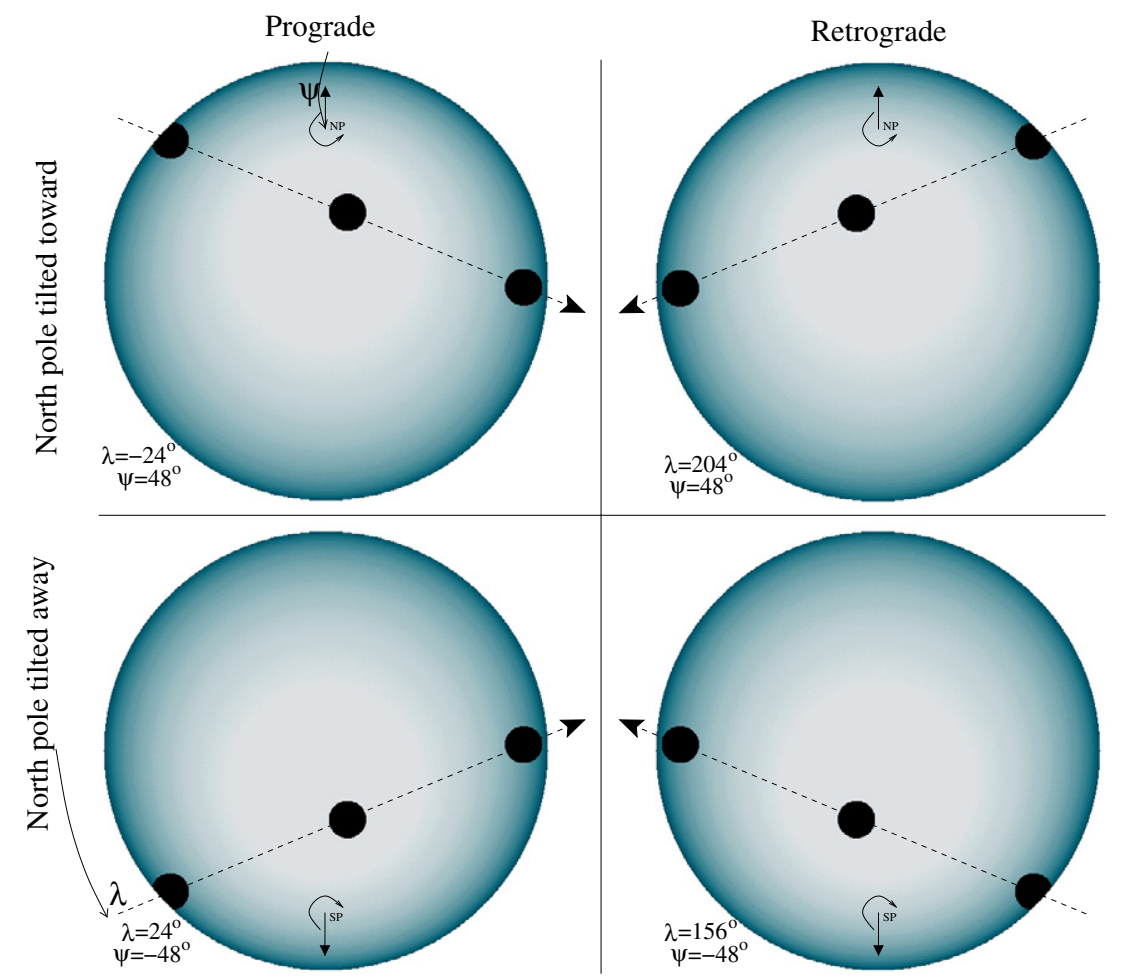

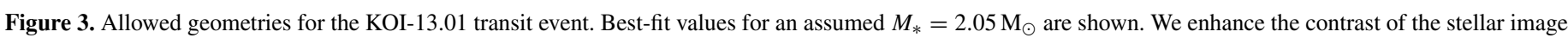

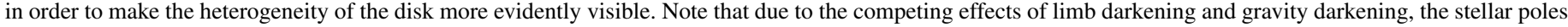

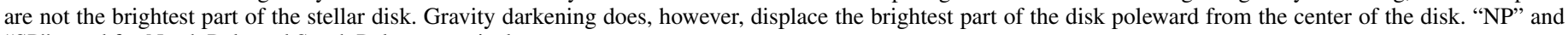
"SP" stand for North Pole and South Pole, respectively.

(A color version of this figure is available in the online journal.)

from inter-transit variability in the Kepler photometer. Although each individual data point from the Kepler Q2 data set has highprecision, red noise on longer timescales leads to systematic errors between transits. Hence, folding the transits introduced a modest amount of additional noise over and above the precision of the individual points.

We adjusted the formal $1 \sigma$ error bars to account for the final $\chi_{\text {reduced }}^{2}$ of each fit. The errors that we indicate in Table 1 represent the formal errors of the fit. They do not include, for instance, likely systematic errors from our assumptions of stellar mass, $T_{\text {pole }}$, the binary flux fraction, and/or fixed $v \sin i$.

Our measured value for the stellar radius that we derive from the lightcurve fit, $R_{*}=1.756 \pm 0.014 \mathrm{R}_{\odot}$, is substantially lower than the spectroscopic value reported by Szabó et al. (2011). Given a similar planet-star radius ratio, then, we also measure a smaller radius for the transiting body, $R_{\mathrm{p}}=1.445 \pm 0.016 \mathrm{R}_{\mathrm{Jup}}$. This smaller radius for the transiter places it within the range of possible planetary companions for relatively young, highly irradiated planets (Fortney et al. 2007). According to Figure 5 from Fortney et al. (2007), both $M_{\mathrm{p}}=1 \mathrm{M}_{J}$ and $M_{\mathrm{p}}=3 \mathrm{M}_{J}$ planets with $25 \mathrm{M}_{\oplus}$ cores could have radii of $R_{\mathrm{p}}=1.44 \mathrm{R}_{\mathrm{Jup}}$ with an age of $700 \mathrm{Myr}$ as determined by Szabó et al. (2011). This raises the possibility that KOI-13.01's mass lies in the planetary regime, not that of brown dwarfs as the higher $R_{\mathrm{p}}$ of Szabó et al. (2011) suggested.

By fitting for both $R_{*}$ and our limb-darkening parameter $c_{1}$, we arrive at a more consistent measure of the planet's orbit inclination relative to the plane of the sky, $i$. Expressed as an impact parameter $b$, our values near $b=0.32$ are significantly lower (i.e., the transit is more nearly central, and less close to grazing) than the value determined by Szabó et al. (2011). The discrepancy may lie in the assumption by Szabó et al. (2011) of a spectroscopically determined stellar radius that was rather higher than our best-fit value. Because the stellar oblateness, $f_{*}$, affects ingress and egress times (similar to the way that planetary oblateness does, see Barnes \& Fortney 2003), our measured impact parameter is more accurate than one that would result from a fit without explicitly incorporating stellar oblateness.

Stellar oblateness $f_{*}$ also affects the measured time of inferior conjunction, $T_{0}$. For rapidly rotating, oblate stars, the mid-transit time and the time of inferior conjunction can differ (Barnes 2009), depending on the transit geometry. The measured times for the fit assuming $M_{*}=1.83 \mathrm{M}_{\odot}(4628032 \pm 3$ $\mathrm{s}$ after BJD 2454900) and that while assuming $M_{*}=2.05 \mathrm{M}_{\odot}$ $(4628033 \pm 3$ s after BJD 2454900) are slightly different because the intensity of the effect depends on the stellar oblateness, which is different in the two fits (see below). Both values are somewhat later than the mid-transit time reported in Borucki et al. (2011), $4628014 \pm 10 \mathrm{~s}$. Since the values are within $2 \sigma$, however, the discrepancy could be due to the stellar oblateness, but may also be statistical variation.

The transit geometry determinations represent the most important measurements that we describe in this paper. Our value for the sky-projected spin-orbit angle $\lambda$ can directly be compared to measurements of $\lambda$ made in other transiting systems from the Rossiter-McLaughlin effect. Unlike Rossiter-McLaughlin measurements, however, we are also able to determine the stellar obliquity, $\psi$, which we define as the amount by which the star's north pole is tipped out of the plane of the sky toward the observer.

We cannot determine $\lambda$ and $\psi$ uniquely, though. We determine four allowed transit geometries, as diagrammed in Figure 3. For 
the $M_{*}=2.05 \mathrm{M}_{\odot}$ case, those are (clockwise from lower left): (1) $\lambda=24^{\circ}, \psi=-48^{\circ}$, the prograde case with north pole tipped away from the observer; (2) $\lambda=-24^{\circ}, \psi=48^{\circ}$, the prograde case with the north pole tipped toward the observer; (3) $\lambda=-156^{\circ}, \psi=48^{\circ}$, the retrograde case with the north pole tipped toward the observer; and (4) $\lambda=156^{\circ}, \psi=-48^{\circ}$, the retrograde case with the north pole tipped away from the observer.

The combination of $\lambda$ and $\psi$, along with the planet's orbit inclination $i$, allows us to calculate the complete relative angle between the stellar rotation pole and the planetary orbit normal — the spin-orbit alignment, $\varphi$ —using Winn et al. (2007), Equation (7). With our angle definitions, this equation becomes

$$
\cos \varphi=\sin \psi \cos i+\cos \psi \sin i \cos \lambda .
$$

We measure $\varphi=54^{\circ} \pm 4^{\circ}$ for $M_{*}=1.83 \mathrm{M}_{\odot}$ and $\varphi=56^{\circ} \pm 4^{\circ}$ for $M_{*}=2.05 \mathrm{M}_{\odot}$. Hence the orbiting body, KOI-13.01, is far from spin-orbit alignment. While the projected alignment $\lambda$ (as would be measured using the Rossiter-McLaughlin effect) alone reveals a significant deviation from alignment, the full extent of the misalignment manifests from the large stellar obliquity $\psi$ which would not be measured by the Rossiter-McLaughlin effect (i.e., Fabrycky \& Winn 2009).

In actuality, given the orbital geometry degeneracies described by Figure 3, $\varphi$ also has another solution-one where the planet orbits retrograde. In that case, $\varphi=126^{\circ} \pm 4^{\circ}$ for $M_{*}=1.83 \mathrm{M}_{\odot}$ and $\varphi=124^{\circ} \pm 4^{\circ}$ for $M_{*}=2.05 \mathrm{M}_{\odot}$. We offer a possibility for resolving the prograde/retrograde degeneracy photometrically in Section 5.

\section{DISCUSSION}

\subsection{Implications}

Our determination of a high spin-orbit misalignment for KOI-13.01, $\sim 55^{\circ}$ rather than the solar system's more typical $\leqslant 6^{\circ}$, drives us to pose the question of how the spin-orbit misalignment came to be. Assuming that KOI-13.01 formed like a solar system planet, in a planar disk orbiting the protostellar KOI-13, there are two ways to have developed a spin-orbit misalignment: either the stellar spin axis changed, or the planet's orbit did (or both). In addition to explaining the spin-orbit misalignment of KOI-13.01, any explanation for its formation and evolution must also explain KOI-13.01's apparently circular orbit, as established by the presence of a properly timed secondary eclipse (Szabó et al. 2011).

Bate et al. (2010) suggest a mechanism by which to alter the star's rotation axis, moving it away from the normal to the protoplanetary disk. This mechanism could drive spin-orbit misalignment and allows for KOI-13.01 to end up on a circular orbit.

Two prominent ways to alter the planet's orbit are by planet-planet scattering and the Kozai mechanism. If a planet-planet scattering event occurred early enough in the planet formation process, then sufficient debris might have remained to circularize the orbit before the disk dissipated.

A Kozai-driven source for spin-orbit misalignment, like that for HD 80606b (Wu \& Murray 2003; Winn et al. 2009a), is appealing because of the presence of KOI-13 's binary companion. However, this mechanism requires that the planet's orbital precession due to the binary companion be more rapid than that induced by the planet's parent star alone. Wu \& Murray (2003) suggest that HD80606b's Kozai variations thus ended when its orbital semimajor axis dropped, reducing Kozai's effectiveness while increasing the effects from its parent star.

For this mechanism to be viable for the evolution of KOI-13.01, its orbit would have had to have been circularized from a presumed prior highly eccentric Kozai-derived orbit. Given KOI-13 's young 700 Myr age, though, tidal circularization would seem difficult. The conventional tidal circularization timescale $\tau_{\mathrm{c}}=\frac{e}{d e / d t}$ (Trilling 2000) for KOI-13.01 in its present state is $\tau_{\mathrm{c}}=\sim 8 \mathrm{Myr}$. However in a presumed former state like that of HD80606b presently, with a semimajor axis of $0.5 \mathrm{AU}$, the circularization timescale is $\tau_{\mathrm{c}}=200$ trillion years using the value for $M_{\mathrm{p}}$ from Shporer et al. (2011b). Hence, we think that the Kozai mechanism is likely not responsible for the spin-orbit misalignment of KOI-13.01, since the end state of Kozai migration would be a highly eccentric orbit that could not have been circularized in the star's lifetime. A more complete numerical study of the orbital circularization of KOI-13.01, tracking the coupled evolution of both eccentricity and semimajor axis following Jackson et al. (2008), might shed additional light on the issue.

\subsection{Future Work}

The present work used just the public Q2 short-cadence Kepler data for KOI-13. Presuming that Kepler continues to observe KOI-13 at short cadence, the ultimate coadded photometric precision should continue to improve. The resulting higher signal-to-noise ratio will allow more precise determinations of all of the transit parameters in Table 1. As the precise shape of the asymmetry at the bottom of the lightcurve becomes clearer, the purely photometric determination of the star's mass $M_{*}$ should approve commensurately.

Another effect that will become important as precision improves is the photometric Rossiter-McLaughlin effect (Shporer et al. 2011a; Groot 2011) - the photometric analog of the Rossiter-McLaughlin effect. This effect arises due to the rotation of the star combined with the beaming effect. During transit the planet covers up varying parts of the star, with different apparent radial velocities. The different radial velocities cause photons emitted from different parts of the stellar surface to be beamed by a different amount, resulting in an anomalous photometric signal during transit. This combined effect has been proposed to investigate the spin-orbit alignment of Kepler's binary star population (Shporer et al. 2011a).

We show a calculation of the intensity of this effect for KOI-13.01 in Figure 4. While its present amplitude of $\sim 4 \mathrm{ppm}$ renders it undetectable in the present Q2 Kepler data, future improvements in precision as the mission progresses will make the effect detectable and more easily discernible from the gravity-darkening effects addressed in this paper. Most importantly, the sign of the photometric Rossiter-McLaughlin effect would be flipped for retrograde-orbiting planets. Hence, the photometric Rossiter-McLaughlin effect holds promise in the future for a purely photometric resolution to the prograde/retrograde degeneracy that we show in Figure 3.

Finally, Barnes (2009) showed that the lightcurve shape varies for spin-orbit misaligned planets when viewed at differing wavelengths. We show an illustration of this effect for KOI13.01 in Figure 5. Because the intensity of the difference between the lightcurve in the Kepler bandpass and that at different wavelengths depends on $T_{\text {pole }}$, such observations could serve to place constraints on $T_{\text {pole }}$. 


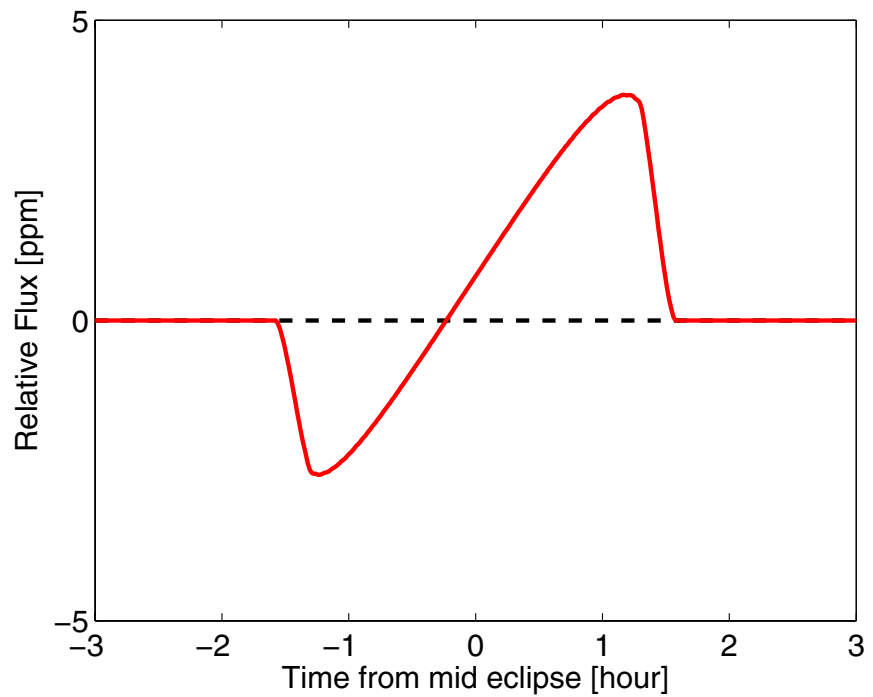

Figure 4. This plot shows the intensity of the photometric Rossiter-McLaughlin effect for KOI-13.01 using our best-fit parameters for $M_{*}=2.05 \mathrm{M}_{\odot}$ from Table 1. While our algorithm presently does not account for this effect, it could be incorporated in the future as additional Kepler data improve the period-folded photometric precision. If it could be detected, the photometric Rossiter-McLaughlin effect could be used to break the prograde/retrograde degeneracy for the planet's orbit depicted in Figure 3.

(A color version of this figure is available in the online journal.)

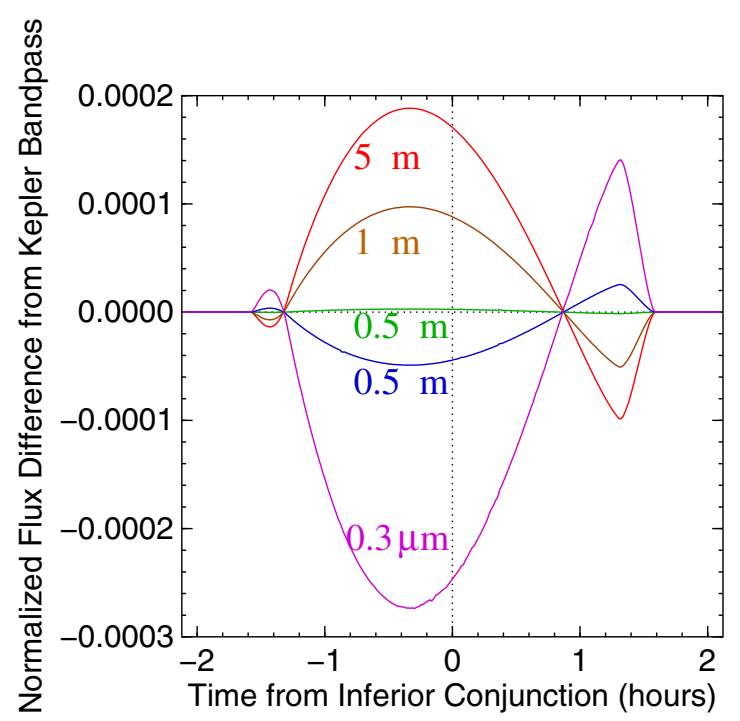

Figure 5. Here we show the difference between various theoretical lightcurves, conforming to the transit parameters from Table 1, acquired at various indicated monochromatic wavelengths relative to that integrated across the Kepler bandpass. At shorter wavelengths the KOI-13.01 transit lightcurve asymmetry is increased, and at longer wavelengths the asymmetry is more muted. A singlewavelength lightcurve at $0.5934 \mu \mathrm{m}$ most closely replicates that of the lightcurve under the entire Kepler bandpass for KOI-13. Observation of this transit, if sufficiently precise, could constrain $T_{\text {pole }}$ and the gravity-darkening parameter $\beta$.

(A color version of this figure is available in the online journal.)

\section{CONCLUSION}

Barnes (2009) first discussed the prospect for spin-orbit misaligned planets orbiting rapidly rotating stars having asymmetric transit lightcurves owing to stellar gravity darkening (von Zeipel 1924). Szabó et al. (2011) showed that planet candidate KOI-13.01, first identified in Kepler data by Borucki et al. (2011), has an asymmetric transit lightcurve. Szabó et al. (2011) also made new critical measurements of KOI-13, including the binary flux fraction and $v \sin i$ from stellar rotation.

Fitting the Q2 short cadence Kepler photometry for KOI-13.01 with the Barnes (2009) model, we measured a projected spin-orbit alignment $\lambda=23^{\circ} \pm 4^{\circ}$ and a stellar obliquity (defined as the angle by which the stellar north pole is tilted out of the plane of the sky toward the observer) of $\psi=-48^{\circ} \pm 4^{\circ}$. While these measurements assume $M_{*}=2.05 \mathrm{M}_{\odot}$ as determined by Szabó et al. (2011), other fits using different assumed stellar masses show that the overall spin-orbit misalignment derived for the star, $\varphi=56^{\circ} \pm 4^{\circ}$, is not substantially affected by plausible stellar mass variations. The photometric determination also allows for a retrograde orbit with $\varphi=124^{\circ} \pm 4^{\circ}$. Our measurement of $\varphi$ is the first spin-orbit determination to come from gravity darkening. It is one of just a handful of observations of any kind to determine the complete spin-orbit misalignment of a transiting planetary-class body, not just its projected spin-orbit alignment $\lambda$ (Winn et al. 2007; Sanchis-Ojeda \& Winn 2011; Nutzman et al. 2011).

The spin-orbit misalignment of KOI-13.01 follows the trend that planets around more massive stars are more likely to be spin-orbit misaligned (Winn et al. 2010a). Our KOI13.01 measurement together with the spin-orbit misaligned WASP-33b around a $M_{*}=1.5 \mathrm{M}_{\odot}$ star (Collier Cameron et al. 2010) extends the Winn et al. (2010a) to yet-higher stellar masses.

We also measure the other critical transit parameters for the KOI-13.01 system. We determined $R_{*}=1.756 \pm 0.014 \mathrm{R}_{\odot}$, $R_{\mathrm{p}}=1.445 \pm 0.016 \mathrm{R}_{\mathrm{Jup}}, i=85.9 \pm 0.4, b=0.31598$, and a single limb-darkening parameter of $c_{1}=0.48 \pm 0.03$. Our photometrically determined stellar and planetary radii are much lower than those of Szabó et al. (2011) who used a spectroscopically derived star radius of $2.44 \mathrm{R}_{\odot}$. Our lower estimated radius places KOI-13.01 in the regime where interior models allow for highly irradiated planetary-mass objects (Fortney et al. 2007).

We also take advantage of the dependence of gravity darkening on the stellar surface gravity to directly measure $M_{*}$ from the Kepler transit lightcurve. Transit lightcurves for spherical stars can only constrain the stellar density $\rho_{*}$ and do not provide any absolute length scale with which to constrain $M_{*}$. Smaller stars would need to rotate faster to provide the measured $v \sin i=65 \mathrm{~km} \mathrm{~s}^{-1}$, and thus they drive more intense curvature at the bottom of the lightcurves of transiting objects. Conversely, more massive stars have correspondingly lower gravity darkening, and less lightcurve asymmetry. The effect is weak enough that our highly uncertain measurement of $M_{*}=0.9 \pm 0.6 \mathrm{M}_{\odot}$ cannot differentiate between the Borucki et al. (2011) and Szabó et al. (2011) spectroscopic measurements of $M_{*}=1.83 \mathrm{M}_{\odot}$ and $M_{*}=2.05 \mathrm{M}_{\odot}$ respectively. While it is consistent with (or within $2 \sigma$ of) either measurement, and inconsistent with the measured spectral type (A), we do weakly prefer lower values of $M_{*}$. Future observations, if more precise, can help to more tightly constrain this first-ever measurement of $M_{*}$ from gravity darkening.

Additional Kepler photometry may also show evidence for the photometric Rossiter-McLaughlin effect (Shporer et al. 2011a; Groot 2011) in the KOI-13.01 transit lightcurve. This sign of this effect, if it can be measured, would resolve the degeneracy between prograde and retrograde planetary orbits using photometry alone.

We have not determined the cause of KOI-13.01's spin-orbit misalignment. However, we note that the Kozai mechanism, implicated for the evolution of HD80606b (Wu \& Murray 2003), 
may be inconsistent with KOI-13.01's circular orbit according to a simple analysis, despite the presence of a similar-mass binary companion to KOI-13. A more sophisticated study of the effectivity of the Kozai mechanism for KOI-13.01 can help to resolve the origin of the spin-orbit misalignment. Stellar spin drift and planet-planet scattering during formation remain viable misalignment generation mechanisms.

The complex and interesting nature of the KOI-13.01 star-planet system make it an astrophysical laboratory for photometric transit effects. The exquisite photometric precision of the Kepler photometer allows us to probe those effects for the first time. The lessons that we learn here will then be applicable to future transiting objects discovered by Kepler and others.

\section{REFERENCES}

Albrecht, S., Reffert, S., Snellen, I. A. G., Quirrenbach, A., \& Mitchell, D. S. 2007, A\&A, 474, 565

Albrecht, S., Reffert, S., Snellen, I. A. G., \& Winn, J. N. 2009, Nature, 461, 373

Albrecht, S., Winn, J. N., Carter, J. A., Snellen, I. A. G., \& de Mooij, E. J. W. 2011, ApJ, 726, 68

Anderson, D. R., Hellier, C., Gillon, M., et al. 2010, ApJ, 709, 159

Barnes, J. W. 2009, ApJ, 705, 683

Barnes, J. W., \& Fortney, J. J. 2003, ApJ, 588, 545

Bate, M. R., Lodato, G., \& Pringle, J. E. 2010, MNRAS, 401, 1505

Borucki, W. J., Koch, D., Basri, G., et al. 2010, Science, 327, 977

Borucki, W. J., Koch, D. G., Basri, G., et al. 2011, ApJ, 736, 19

Brown, T. M., Charbonneau, D., Gilliland, R. L., Noyes, R. W., \& Burrows, A. 2001, ApJ, 552, 699

Brown, T. M., Latham, D. W., Everett, M. E., \& Esquerdo, G. A. 2011, AJ, 142, 112

Collier Cameron, A., Guenther, E., Smalley, B., et al. 2010, MNRAS, 407, 507

Cox, A. N., (ed.) 2000, Allen's Astrophysical Quantities (4th ed.; New York: AIP)

Fabrycky, D. C., \& Winn, J. N. 2009, ApJ, 696, 1230

Fortney, J. J., Marley, M. S., \& Barnes, J. W. 2007, ApJ, 659, 1661

Groot, P. J. 2011, ApJ, in press (arXiv:1104.3428)

Hébrard, G., Bouchy, F., Pont, F., et al. 2009, in IAU Symp. 253, Transiting Planets, ed. F. Pont, D. Sasselov, \& M. Holman (Cambridge: Cambridge Univ. Press), 508
Hellier, C., Anderson, D. R., Collier-Cameron, A., et al. 2011, ApJ, 730, L31

Hirano, T., Narita, N., Shporer, A., et al. 2011, PASJ, 63, 531

Jackson, B., Greenberg, R., \& Barnes, R. 2008, ApJ, 678, 1396

Johnson, J. A., Winn, J. N., Bakos, G. Á., et al. 2011, ApJ, 735, 24

Johnson, J. A., Winn, J. N., Narita, N., et al. 2008, ApJ, 686, 649

Kipping, D. M. 2010, MNRAS, 408, 1758

Lissauer, J. J. 1993, ARA\&A, 31, 129

Lissauer, J. J., Fabrycky, D. C., Ford, E. B., et al. 2011, Nature, 470, 53

Mandel, K., \& Agol, E. 2002, ApJ, 580, L171

Miller, G. R. M., Collier Cameron, A., Simpson, E. K., et al. 2010, A\&A, 523, A52

Monnier, J. D., Zhao, M., Pedretti, E., et al. 2007, Science, 317, 342

Murray, C. D., \& Dermott, S. F. 2000, Solar System Dynamics (Cambridge: Cambridge Univ. Press)

Narita, N., Sato, B., Hirano, T., \& Tamura, M. 2009, PASJ, 61, L35

Nutzman, P. A., Fabrycky, D. C., \& Fortney, J. J. 2011, ApJ, 740, L10

Pál, A. 2008, MNRAS, 390, 281

Perryman, M. 2011, The Exoplanet Handbook (Cambridge: Cambridge Univ. Press)

Press, W. H., Teukolsky, S. A., Vetterling, W. T., \& Flannery, B. P. 2007, Numerical Recipes: The Art of Scientific Computing (3rd ed.; Cambridge: Cambridge Univ. Press)

Rowe, J., Borucki, W. J., Howell, S. B., et al. 2011, BAAS, 217, 103.04

Sanchis-Ojeda, R., \& Winn, J. N. 2011, ApJ, in press (arXiv:1107.2920)

Sanchis-Ojeda, R., Winn, J. N., Holman, M. J., et al. 2011, ApJ, 733, 127

Seager, S., \& Hui, L. 2002, ApJ, 574, 1004

Seidelmann, P. K. 1992, Explanatory Supplement to the Astronomical Almanac (Mill Valley, CA: Univ. Science Books)

Shporer, A., Brown, T., Mazeh, T., \& Zucker, S. 2011a, arXiv:1107.4458

Shporer, A., Jenkins, J. M., Rowe, J. F., et al. (Kepler Science Team) 2011b, AJ, submitted (arXiv:1110.3510)

Simpson, E. K., Pollacco, D., Cameron, A. C., et al. 2011, MNRAS, 414, 3023

Simpson, E. K., Pollacco, D., Hébrard, G., et al. 2010, MNRAS, 405, 1867

Szabó, G. M., Szabó, R., Benkő, J. M., et al. 2011, ApJ, 736, L4

Triaud, A. H. M. J., Queloz, D., Bouchy, F., et al. 2009, A\&A, 506, 377

Trilling, D. E. 2000, ApJ, 537, L61

von Zeipel, H. 1924, MNRAS, 84, 665

Winn, J. N., Fabrycky, D., Albrecht, S., \& Johnson, J. A. 2010a, ApJ, 718, L145

Winn, J. N., Holman, M. J., Henry, G. W., et al. 2007, AJ, 133, 1828

Winn, J. N., Howard, A. W., Johnson, J. A., et al. 2009a, ApJ, 703, 2091

Winn, J. N., Johnson, J. A., Albrecht, S., et al. 2009b, ApJ, 703, L99

Winn, J. N., Johnson, J. A., Howard, A. W., et al. 2010b, ApJ, 723, L223

Winn, J. N., Johnson, J. A., Marcy, G. W., et al. 2006, ApJ, 653, L69

Wu, Y., \& Murray, N. 2003, ApJ, 589, 605 